Global Program for the Elimination of Silicosis. In 2006, at the second international meeting of International Plan for Americas, a proposal to build a map of exposure situation based on a Job of Potential Exposure Matrix (JPEM) to silica as a surveillance methodology was made.

Objectives Estimate the numbers of workers exposed to silica in Brazil, Chile, Venezuela, Peru and Colombia.

Methods A JPEM developed in Brazil and matched with the study in Chile, according to the conception of CAREX database, was applied to workers in Chile, Venezuela, Peru and Colombia.

Results The percentages of workers exposed to crystalline silica were: Brazil (5.6\%), Chile (5.4\%), Peru (4.9\%), Venezuela (5.2\%) and Colombia $(6.0 \%)$. When compare to the percentage of workers exposed in Finland (3.8\%), Spain (3.3\%), Germany (2.9\%) Great Britain $(2.6 \%)$ and Italy $(1.6 \%)$, these data shows the relevance of silica dust exposure in the countries with economies based on polluting and health damaging technologies. Historically, this inequality was given by the transfer of obsolete technology and harmful work activities.

Conclusion Traditional surveillance, based only on the identification of cases, does not identify this historic process. Thus, job-exposure matrices are a potentially valuable addition to epidemiologic research methods. If applied judiciously, may contribute to etiologic research and to the identification and control of workplace exposures.

\section{P1-304 ASSOCIATION BETWEEN QUALITY OF LIFE AND NUTRITION STATUS OF OLDER ADULTS IN A MEDIUM- SIZED CITY IN SÃO PAULO STATE, BRAZIL}

doi:10.1136/jech.2011.142976e.96

${ }^{1} \mathrm{~L}$ B de Souza, ${ }^{1} \mathrm{~J}$ E Corrente.* ${ }^{1}$ Graduate Program in Collective Health, Botucatu School of Medicine, UNESP, Botucatu, São Paulo, Brazil; ${ }^{2}$ Department of Biostatistics, Biosciences Institute, UNESP, Botucatu, São Paulo, Brazil

Introduction In the last few decades, population growth patterns have shown high figures for older adults. Accentuated increase in the number of older individuals, particularly in developed countries, has brought consequences to society, and in order to face such challenge, it is necessary to identify the determinant causes of older persons' present health and life conditions.

Objective This study aimed at evaluating the existence of an association between quality of life and nutritional status in a sample of older residents in the city of Botucatu - São Paulo, Brazil.

Methodology It was an epidemiological, cross-sectional, populationbased study on individuals aged 60 years or older. A home interview was conducted with 96 elders. Their anthropometric measures were obtained and a Flanagan Quality of Life Scale (FOOLS) was applied.

Results The older individuals' mean age individuals was $74.1 \pm 7$ years. Most of them were females (60\%), poorly educated, married $(62.11 \%)$ and retired (84.21\%). According to FOOLS, $72.62 \%$ reported to be satisfied about their quality of life. As to nutritional status, it was found that $41 \%$ of the participants were overweight. Anthropometric measurements reduced as age advanced, although such reduction was not always significant. No significant association was observed between anthropometric measurements or nutritional status and quality of life.

Conclusions Although the majority of the older people reported to have good quality of life, obesity is still a factor of concern at this age range, and nutritional intervention programs as well as incentive to healthy diets should be recommended. P1-305 ROLE OF PROXIMAL AND DISTAL DETERMINANTS IN HAND
WASHING AND WATER TREATMENT PRACTICES IN SLUMS OF INDIA

doi:10.1136/jech.2011.142976e.97

Dr Ritvik, ${ }^{*}$ R A Krishnan, S Kapoor, C Pandav. All India institute of medical sciences, New Delhi, India

Introduction The determinants of health and disease can be classified as proximal and distal depending on their position in the causal pathway. This study attempts find out whether behaviour change communication interventions targeted at the proximal determinants (knowledge and infrastructure) of handwashing and water treatment change the relative importance of distal socio-economic status determinants.

Methods The analysis uses data from an evaluation of a Safe Water System intervention in urban slums of Haridwar and Dehradun districts of Uttarakhand, India. As a part of evaluation, two independent surveys were conducted in the SWS intervention area in $2004(n=1125)$ and $2005(n=1128)$. Analysis was based on framework of hierarchical modelling. Logistic regression was applied and the change in "population attributable fraction" (PAF) from baseline to endline models was calculated for arriving at the relative importance of the determinants.

Results At baseline, distal determinants were important for water treatment. Introduction of software variables to the model resulted in decline in the PAF of distal determinants from $89 \%$ to $74 \%$. At endline, the importance of distal determinants was further reduced (PAF of 23\%). For handwashing at baseline, the distal variables were less important for handwashing (PAF of 36\%). After intervention, there was smaller decline in their importance (PAF of $22.7 \%$ ). Proximal determinants assumed importance only after intervention. The models in the study could only partially explain the variance in two behaviours under study.

Conclusions Behaviour change communication interventions can help overcome influence of low socio-economic status or illiteracy by changing proximal determinants.

\section{P1-306 THE INFLUENCE OF CHILDHOOD DEVELOPMENTAL PLASTICITY AND SOCIOECONOMIC CIRCUMSTANCES ON ADULT HEALTH BIOMARKERS: LONGITUDINAL EVIDENCE FROM THE WEST OF SCOTLAND TWENTY-07 STUDY}

doi:10.1136/jech.2011.142976e.98

T Robertson, ${ }^{*}$ M Benzeval. Medical Research Council, Social \& Public Health Sciences Unit, Glasgow, UK

Physical development in early life adapts to environmental circumstances through developmental plasticity. Despite short-term gains, altered developmental trajectories can have long-term health consequences. This study investigates the individual and combined associations of childhood socioeconomic circumstances and developmental plasticity with adult health biomarkers. Respondents from the West of Scotland Twenty-07 Study were followed from ages 15 to 35 , with biomarkers sampled at 35 $(n=736)$. Social class mobility from birth to 15 categories were stable non-manual, stable manual, upwardly mobile or downwardly mobile. Developmental categories, based on birthweight and height at 15 tertiles, were stable (eg, average birthweight and height), compensatory (eg, low birthweight, tall height) or limited (eg, large birthweight, short height). Sex-specific GLMs including developmental and socioeconomic categories (separate and combined models) were analysed against Forced Expiratory Volume in $1 \mathrm{~s}$ 
$\left(\mathrm{FEV}_{1}\right)$, HDL-cholesterol (HDL), body mass index (BMI), systolic blood pressure (BP), and glycated haemoglobin (HbA1c). In men, those in stable manual and upward (vs stable non-manual) class categories showed higher BMI, HbA1c and BP, and lower HDL and $\mathrm{FEV}_{1} \quad(\mathrm{p}<0.10)$. Limited development was associated with higher BMI and $\mathrm{HbA1c}$, and lower $\mathrm{FEV}_{1}(\mathrm{p}<0.05)$. In women, those in the stable manual class had lower HDL and $\mathrm{FEV}_{1}$, and higher HbA1c $(p<0.05)$. Downward mobility was associated with lower HDL $(p=0.018)$. Compensatory development was associated with higher $\mathrm{FEV}_{1}(\mathrm{p}<0.05)$. In both sexes, the socioeconomic- and developmental plasticity- biomarker associations remained significant in the combined analysis. Socioeconomic circumstances and developmental plasticity were associated with negative biomarker outcomes, although they follow independent associative pathways.

\section{P1-307 HIGHER CANCER DETECTION RISK IN WOMEN WITH A FALSE POSITIVE RESULT IN BREAST CANCER SCREENING IN SPAIN}

doi:10.1136/jech.2011.142976e.99

\begin{abstract}
${ }^{1} \mathrm{~J}$ Blanch, ${ }^{1} \mathrm{~A}$ Romero, ${ }^{1} \mathrm{M}$ Sala, ${ }^{1} \mathrm{R}$ Román, ${ }^{*}{ }^{2} \mathrm{D}$ Salas, ${ }^{3} \mathrm{R}$ Zubizarreta, ${ }^{4} \mathrm{M}$ Ederra, ${ }^{5} \mathrm{M}$ Baré, ${ }^{6} \mathrm{~J}$ Galceran, ${ }^{7} \mathrm{I}$ González-Román, ${ }^{8} \mathrm{C}$ Natal, ${ }^{9} \mathrm{M}$ de la Vega, ${ }^{10} \mathrm{~A}$ Baroja, ${ }^{1} \mathrm{~F}$ Macià, ${ }^{1} \mathrm{X}$ Castells. ${ }^{1}$ Department of Epidemiology and Evaluation, IMIM-Parc de Salut Mar, CIBERESP, Barcelona, Spain; ${ }^{2}$ General Directorate Public Health \& Centre for Public Health Research, Valencia, Spain; ${ }^{3}$ Galician breast cancer screening programme. Public health \& Planning Directorate. Health Office, Santiago de Compostela, Spain; ${ }^{4}$ Navarra Breast Cancer Screening Programme. Public Health Institute, CIBERESP, Pamplona, Spain; ${ }^{5}$ OTC-Epidemiology, Sabadell, Spain; ${ }^{6}$ PDPCM, League Foundation and Cancer Prevention Research, IISPV, Reus, Spain; ${ }^{7}$ Castilla-Leon Breast Cancer Screening Programme, D G Salud Pública ID e I, SACYL, Valladolid, Spain; ${ }^{8}$ Unit of Analysis and Programs. Health Service of Asturias, Oviedo, Spain; ${ }^{9}$ General Directorate of Health Care Programmes. Canary Islands Health Service, Santa Cruz de Tenerife, Spain; ${ }^{10}$ La Rioja Breast Cancer Screening Programme, Fundacion Rioja Salud, Logroño, Spain
\end{abstract}

Introduction Breast screening reduces mortality from breast cancer through early detection. Despite its benefits, breast cancer screening presents some adverse effects, as false positive (FP) results. Our aim was to estimate the risk of cancer detection associated to have experienced a previous FP mammography.

Methods The Cumulative False Positive Risk study includes eight population-based screening programs in Spain, between 1990 and 2006, that invites women aged between $45 / 50$ and 69 years, with no previous breast cancer. We included women participating in at least two screenings rounds. A positive mammogram reading was considered a FP result if, after further assessments, breast cancer was not diagnosed. Cancer detection risk was estimated through $\mathrm{OR}$ with a multivariate discrete-time-hazard model with a random intercept. The model included adjusting variables related to screening programs (radiologic unit, reading method and number of projections) and woman (age, hormone replacement therapy use, menopausal status, previous invasive procedures and familial history of breast cancer).

Results Women who had experienced at least one previous FP result had a higher cancer detection rate than those without a FP (4.72 per 1000 mammograms vs 2.56 per 1000 mammograms). The adjusted $\mathrm{OR}$ of cancer detection was higher in women with a FP in any previous screening $(\mathrm{OR}=1.8895 \% \mathrm{CI} 1.76$ to 2.00$)$.
Conclusion Women with a previous FP result had a higher risk of cancer detection. This result may suggest that factors related to FP could provide useful information to redesign different early detection strategies for specific subgroups of women.

\section{P1-308 EFFECT OF FALSE-POSITIVES AND WOMEN'S CHARACTERISTICS ON THE LONG-TERM ATTITUDE TOWARDS BREAST CANCER SCREENING}

doi:10.1136/jech.2011.142976e.100

${ }^{1} \mathrm{R}$ Román, ${ }^{* 1} \mathrm{M}$ Sala, ${ }^{2} \mathrm{R}$ Zubuzarreta, ${ }^{3}$ Delfrade, ${ }^{4} \mathrm{D}$ Salas, ${ }^{5} \mathrm{M} \mathrm{D}$ la vega, ${ }^{6} \mathrm{~A}$ Baroja, ${ }^{7} \mathrm{C}$ Natal, ${ }^{1} \mathrm{~F}$ Macià, ${ }^{8} \mathrm{M}$ Baré, ${ }^{9} \mathrm{~J}$ Galceran, ${ }^{10}$ I González-Román, ${ }^{1} \mathrm{X}$ Castells. ${ }^{1}$ Department of Epidemiology and Evaluation, IMIM-Parc de Salut Mar, CIBERESP, Barcelona, Spain; ${ }^{2}$ Galician breast cancer screening programme, Public health \& Planning Directorate, Health Office, Santiago de Compostela, Spain; ${ }^{3}$ Navarra Breast Cancer Screening Programme, Public Health Institute, CIBERESP, Pamplona, Spain; ${ }^{4}$ General Directorate Public Health \& Centre for Public Health Research, Valencia, Spain; ${ }^{5}$ General Directorate of Health Care Programmes, Canary Islands Health Service, Santa Cruz de Tenerife, Spain; ${ }^{6}$ La Rioja Breast Cancer Screening Programme. Fundacion Rioja Salud, Logroño, Spain; ' Unit of Analysis and Programs, Health Service of Asturias, Oviedo, Spain; ${ }^{8}$ Epidemiology and Assessment Unit UDIAT-Diagnostic Center, Corporació Parc Taulí, Sabadell, Spain; ${ }^{9}$ PDPCM, League Foundation and Cancer Prevention Research, IISPV, Reus, Spain; ${ }^{10}$ Castilla-Leon Breast Cancer Screening Programme, D.G. Salud Publica ID e I, SACYL, Valladolid, Spain

Introduction Adherence to breast cancer screening is affected by presence of previous false-positives and regular participation in previous invitations. Our aim was to estimate the long-term adherence to breast cancer screening and how false-positive and women's characteristics affect the probability of re-attending screening.

Methods Retrospective Cohort study of women aged 45-69 years invited to participate in any of 10 Spanish breast cancer screening programs between 1990 and 2006. Discrete time hazard models were used to estimate re-attendance probabilities, and to evaluate the effect of false-positives and women's characteristics on re-attendance.

Results We analysed information from 1371218 screened women who underwent 4545346 screening mammograms. The re-attendance probability was $81.7 \%$ (95\% CI 81.63 to 81.76$)$ at first screening, $88.1 \%$ (95\% CI 87.98 to 88.12$)$ at 3rd screening, and $95.6 \%(95 \%$ CI 95.52 to 95.73$)$ at 6 th screening. Women without a false-positive result were more likely to return to the following screening invitation. The re-attendance probability at first screening was $79.3 \%$ (99\% CI 79.0-79.6) and $85.3 \%$ (99\% CI 85.2-85.4) for women with and without a false-positive result, respectively. At sixth screening was $94.6 \%$ (99\% CI 93.8-95.4) and 96.0\% (99\% CI 95.8-96.1), respectively. The factors associated with a higher risk of failing to re-attend the following screening invitation were: age 65-69 years $(\mathrm{OR}=8.48$; $\mathrm{CI}$ 8.31-8.65), missing the first screening invitation $(\mathrm{OR}=1.12$; $\mathrm{CI} 1.11-1.14)$, and previous invasive procedures $(\mathrm{OR}=1.09$; CI 1.07-1.10).

Conclusion False-positive results and other women's characteristics affected the re-attendance to subsequent screening invitations. This information could be useful to provide the maximum available information to women invited to participate and improve compliance in subsequent screening invitations. 